INVITED COMMENTARY

\section{Angiogenesis Review Commentary}

\author{
F. Charles Brunicardi ${ }^{1,2}$
}

Published online: 31 March 2017

(C) Société Internationale de Chirurgie 2017

The authors of this erudite paper are to be congratulated on a beautifully written paper on the origins and conception of the neologism "angiogenesis"; the paper is particularly enjoyable to read as it attempts to uncover the truth behind who legitimately coined "angiogenesis" [1]. Notably, the paper highlights the seminal contributions of surgeon-scientists to the field. For example, the legendary Scottish surgeon-scientist, Dr. John Hunter, made the initial observation of blood vessel formation. The authors confirm that Dr. Hunter observed the growth of blood vessels in deer antlers. In addition, he was the first to notice the capacity of the circulatory system for self-repair and expansion; he observed that vessels "would appear to have more powers of perfecting themselves, when injured, than any other part of the body; for their use is almost immediate and constant, and it is they which perform the operation of restoration on the other parts, therefore they themselves must first be perfect" [2]. This single observation would serve to birth the field of angiogenesis.

However, the authors demonstrate that Dr. Hunter did not conceive the neologism "angiogenesis," which is the first analysis of its kind, since most sources attribute the conception of the term angiogenesis to Dr. Hunter. Upon deeper analysis of this mystery, the authors conclude that Dr. Joseph Marshall Flint was the first to utilize the term in any published paper.

The authors are to be commended for their superb acknowledgment of the great surgeon-scientist, Dr. Judah

F. Charles Brunicardi

Francis.Brunicardi@utoledo.edu

1 University of Toledo College of Medicine and Life Sciences, Toledo, OH, USA

2 ProMedica Health System, Toledo, OH, USA
Folkman, for his remarkable contributions to the field of angiogenesis and its correlation to cancer. While the scope of the paper pertains mainly to the conception and development of the word "angiogenesis," Dr. Folkman deserves recognition and discussion, as his contributions are pivotal to the founding of the field of angiogenesis research and its relationship to cancer. Dr. Folkman was the first to note a tumor's dependency upon angiogenesis for sustenance and expansion [3]. His phenomenal block of scientific work resulted in more than 25 papers published in Science. Most importantly, Dr.Folkman's theory led to various therapies inhibiting the initial neovascularization of tumors-such as Avastin (2004) and thalidomide (2006) [4]. Dr. Folkman's work ushered in a new era in angiogenesis research and would have certainly won the Nobel Prize; however, his untimely death prevented this deserved recognition.

\section{References}

1. Natale G, Bocci G, Lenzi P (2016) Looking for the word "Angiogenesis" in the history of health sciences: from ancient times to the first decades of the twentieth century. World J Surg. doi:10.1007/s00268-016-3680-1

2. Hunter J (1861) The works of John Hunter. Volume II. Observations in comparative anatomy. John van Voorst, London

3. Folkman J (2001) Angiogenesis-dependent diseases. Semin Oncol 28:536-542

4. Andrew Pollack for the New York Times. February 27, 2004 F.D.A Approves Cancer Drug From Genentech 